\title{
Research Article \\ SER Performance of Large Scale OFDM-SDMA Based Cognitive Radio Networks
}

\author{
Mostafa Hefnawi \\ Department of Electrical and Computer Engineering, Royal Military College of Canada, P.O. Box 17000, \\ Station Forces, Kingston, ON, Canada K7K 7B4 \\ Correspondence should be addressed to Mostafa Hefnawi; hefnawi@rmc.ca
}

Received 12 March 2014; Accepted 26 June 2014; Published 21 July 2014

Academic Editor: Ahmed Iyanda Sulyman

Copyright (C) 2014 Mostafa Hefnawi. This is an open access article distributed under the Creative Commons Attribution License, which permits unrestricted use, distribution, and reproduction in any medium, provided the original work is properly cited.

This paper proposes an adaptive large scale multiple input multiple output-beamforming scheme (LSMIMO-BF) for uplink (UL) access in broadband wireless cognitive networks with multiple primary users (PUs) and secondary users (SUs) sharing the same spectrum and employing orthogonal frequency-division multiplexing (OFDM). The proposed algorithm seeks the optimal transmit/receive weight vectors that maximize the UL MIMO channel capacity for each cognitive user while controlling the interference levels to PUs. Under the assumption of very large number of antennas at the base station, a closed-form expression for the symbol error rate (SER) performance of the cognitive LSMIMO-OFDM system is derived and compared with the one based on conventional beamforming schemes such as MIMO-maximum ratio combining (MIMO-MRC). The analysis and simulation show that when SUs are transmitting with the proposed constrained capacity-aware (CCA) scheme, the total interference level at the primary base station is reduced and the SER of PUs is improved compared to the case when the secondary network is using conventional MIMO-MRC. It was also shown that, as the number of base station antennas becomes larger, the constraints imposed by the primary network could be relaxed and the symbol error rate (SER) of SUs is improved without harming the PUs performance.

\section{Introduction}

Recently, we are observing a huge interest in cognitive radio networks (CRNs), from both the research and policy/regulation communities $[1,2]$. These CRNs can intelligently share spectrum and extract more bandwidths via "opportunistic use" of spectrum resources. They will be the essential technology needed to make significantly better use of available spectrum and to address the real issue of fixed spectrum access approaches. However, for CRNs to discover underutilized spectrum and adapt their transmission settings accordingly without causing interference to licensed users, their physical layer needs to be highly flexible and adaptable. Among many possible technologies, orthogonal frequencydivision multiplexing (OFDM) has been widely recognized as a versatile modulation technique that has the potential of fulfilling the requirements of CRNs. Using OFDM it is possible to disable the set of subcarriers used by the primary network and to adaptively change the modulation order according to the channel conditions. However, opportunistic spectrum sharing may not be reliable and may limit the system capacity since it suffers from the interruptions imposed by the primary network (PN) on the secondary network (SN) which must leave the licensed channel when primary users (PUs) emerge. Also, with opportunistic spectrum sharing, secondary users (SUs) can still cause interference to PUs due to their imperfect spectrum sensing. One way to overcome these limitations is to incorporate space division multiple access (SDMA) into OFDM-based CRNs. SDMA can help in achieving higher spectral efficiency, by multiplexing multiple users on the same time-frequency resources. OFDM-SDMA techniques have been successfully deployed in 3G/4G cellular systems based on traditional static spectrum access approach [3-9] and a vast number of multiuser detection algorithms, such as maximum ratio combining (MRC) and minimum meansquared error (MMSE), are presently being tailored towards solving the SDMA processing in MIMO cognitive networks [10-15], where additional constraints to protect licensed users' QoS are imposed. Within this context, we are extending our recently developed capacity-aware-based OFDM-SDMA 
schemes [9] to cognitive radio by constraining the transmitted power of SUs and controlling the interference level to PUs while enhancing the overall system capacity for each SU. Moreover, to relax the power and interference constraints imposed on SUs without affecting the performance of PUs, we are considering the deployment of a large number of base station (BS) antennas in comparison to the served users' antennas. This concept was initially investigated for cellular networks [16-18], and there is a clear potential in the area of cognitive radio where interference between $\mathrm{PN}$ and $\mathrm{SN}$ is even more problematic. With this large number of antennas, the central limit theorem and the law of large numbers can be applied and close to optimal performances can be achieved with the simplest forms of user detection and beamforming, that is, MRC, and eigen-beamforming (EBF). Due to the nonconvexity of the constrained channel capacity, we propose using the steepest ascent gradient of the cognitive OFDMSDMA channel capacity to iteratively seek the transmitting weight and derive a closed-form expression for the SER of the system. Using the derived expression, we compare the performance of the proposed cognitive capacity-aware (CCA) scheme and the conventional nonconstrained MIMO-MRC (eigen-beamforming is performed at the mobile transmitters and MRC at the receiving base station) in the presence of secondary and primary multiuser access interferences as well as the correlated fading encountered in antenna array systems. Through the analysis and simulation, the impacts of the constraints imposed by the PN on the $\mathrm{SN}$ are also investigated.

\section{System Model}

We consider the UL multiuser access scenario shown in Figure 1, where $L_{s}$ SUs and one secondary base station (SBS) coexist with $L_{p}$ PUs and one primary base station (PBS) via concurrent spectrum access. In both networks, the users and the base stations are equipped with multiple antennas. It is also assumed that both the SBS and the PBS receivers detect independent OFDM data streams from multiple SUs and PUs simultaneously on the same time-frequency resources. Let $\mathbf{x}^{\mathbf{s}}[k]=\left\{x_{1}^{s}, x_{1}^{s}, \ldots, x_{L_{s}}^{s}\right\}$ and $\mathbf{x}^{\mathbf{p}}[k]=\left\{x_{1}^{p}, x_{1}^{p}, \ldots, x_{L_{p}}^{p}\right\}$ denote, respectively, the set of $L_{s}$ SUs signals and $L_{p}$ PUs signals transmitted on each subcarrier, $k=1, \ldots, N_{c}$, where $N_{c}$ denotes the number of subcarriers per OFDM symbol in the system. It is assumed that $x_{i}^{s}$ and $x_{i}^{p}$ are complexvalued random variables with unit power; that is, $E\left[\left\|x_{i}^{s}\right\|^{2}\right]=$ $E\left[\left\|x_{i}^{p}\right\|^{2}\right]=1$. The expression for the array output of the SBS in Figure 1 can be written for each subcarrier as

$$
\mathbf{y}_{\mathrm{SBS}}[k]=\sum_{l_{s}=1}^{L_{s}} \mathbf{H}_{s s, l_{s}}[k] \mathbf{w}_{l_{s}}^{t}[k] \mathbf{x}_{l_{s}}^{s}[k]+\mathbf{n}[k]+\mathbf{I}_{\mathrm{PU}}[k],
$$

where $\mathbf{y}_{\mathrm{SBS}}[k]=\left[y_{1}^{s}[k], y_{2}^{s}[k], \ldots, y_{N_{s}^{r}}^{s}[k]\right]^{T}$ is the $N_{s}^{r} \times$ 1 vector containing the outputs of the $N_{s}^{r}$-element array at the SBS, with $(\cdot)^{T}$ denoting the transpose operation, $\mathbf{H}_{s s, l_{s}}[k]$ is the $N_{s}^{r} \times N_{s}^{t}$ frequency-domain channel matrix representing the transfer functions from secondary user $l_{s}$ 's
$N_{s}^{t}$-element antenna array to the SBS's $N_{s}^{r}$-element antenna array, $\mathbf{w}_{l_{s}}^{t}[k]=\left[w_{l_{s}}^{t}[k], w_{l_{s}}^{t}[k], \ldots, w_{l_{s} N_{s}^{t}}^{t}[k]\right]^{T}$ is the $N_{s}^{t} \times 1$ complex transmit weight vector for SU $l_{s}, l_{s}=1, \ldots, L_{s}$, $\mathbf{n}[k]=\left[n_{1}[k], n_{2}[k], \ldots, n_{N_{r}}[k]\right]^{T}$ is the $N_{s}^{r} \times 1$ complex additive white Gaussian noise vector, and $\mathbf{I}_{\mathrm{PU}}[k]$ represents the interference introduced by PUs at the SBS, given by

$$
\mathbf{I}_{\mathrm{PU}}[k]=\sum_{l_{p}=1}^{L_{p}} \mathbf{H}_{p s, l_{p}}[k] \mathbf{w}_{l_{p}}^{t}[k] \mathbf{x}_{l_{p}}^{p}[k],
$$

where $\mathbf{H}_{p s, l_{p}}[k]$ is the $N_{s}^{r} \times N_{p}^{t}$ channel matrix representing the fading coefficients from PUs to the SBS's $N_{s}^{r}$-element antenna array. On the other hand, the interference seen by the primary base station due to secondary transmission is given by

$$
\mathbf{y}^{s p}[k]=\sum_{l_{s}=1}^{L_{s}} \mathbf{H}_{s p, l_{s}}[k] \mathbf{w}_{l_{s}}^{t}[k] \mathbf{x}_{l_{s}}^{s}[k]
$$

and its corresponding power level can be expressed as

$$
J_{s p}[k]=\sum_{i=1}^{L_{s}} \mathbf{H}_{s p, l_{s}}[k] \mathbf{w}_{l_{s}}^{t}[k] \mathbf{w}_{l_{s}}^{t, H}[k] \mathbf{H}_{s p, l_{s}}^{H}[k],
$$

where $(\cdot)^{H}$ denotes the Hermitian transpose and $\mathbf{H}_{s p, l_{s}}[k]$ is the $N_{p}^{r} \times N_{s}^{t}$ channel matrix representing the fading coefficients from the $l_{s}$ th $\mathrm{SU}$ to the PBS's $N_{p}^{r}$-element antenna array. Assuming the well-known Kronecker correlation structure [19-21], $\mathbf{H}_{s s, l_{s}}[k], \mathbf{H}_{s p, l_{s}}[k]$, $\mathbf{H}_{p s, l_{p}}[k]$, and $\mathbf{H}_{p p, l_{p}}[k]$ can be modeled as $\mathbf{H}_{s s, l_{s}}[k]=$ $\mathbf{R}_{r s}^{1 / 2} \mathbf{H}_{s s, l_{s}}^{\omega}[k] \mathbf{R}_{t s}^{1 / 2}, \mathbf{H}_{s p, l_{s}}[k]=\mathbf{R}_{r p}^{1 / 2} \mathbf{H}_{s p, l_{s}}^{\omega}[k] \mathbf{R}_{t s}^{1 / 2}, \mathbf{H}_{p s, l_{p}}[k]=$ $\mathbf{R}_{r s}^{1 / 2} \mathbf{H}_{p s, l_{p}}^{\omega}[k] \mathbf{R}_{t p}^{1 / 2}$, and $\mathbf{H}_{p p, l_{p}}[k]=\mathbf{R}_{r p}^{1 / 2} \mathbf{H}_{p p, l_{s}}^{\omega}[k] \mathbf{R}_{t p}^{1 / 2}$, respectively, where $\mathbf{R}_{r s} \in \mathbb{C}^{N_{s}^{r} \times N_{s}^{r}}, \mathbf{R}_{r p} \in \mathbb{C}^{N_{p}^{r} \times N_{p}^{r}}, \mathbf{R}_{t s} \in$ $\mathbb{C}^{N_{s}^{t} \times N_{s}^{t}}$, and $\mathbf{R}_{t p} \in \mathbb{C}^{N_{p}^{t} \times N_{p}^{t}}$ are positive definite Hermitian matrices specifying the receive and transmit fading correlations. $\mathbf{H}_{s s, l_{s}}^{\omega}[k] \in \mathbb{C}^{N_{s}^{r} \times N_{s}^{t}}, \mathbf{H}_{s p, l_{s}}^{\omega}[k] \in \mathbb{C}^{N_{p}^{r} \times N_{s}^{t}}$, $\mathbf{H}_{p s, l_{p}}^{\omega}[k] \in \mathbb{C}^{N_{s}^{r} \times N_{p}^{t}}$, and $\mathbf{H}_{p p, l_{s}}^{\omega}[k] \in \mathbb{C}^{N_{p}^{r} \times N_{p}^{t}}$ consist of iid (independent and identically distributed) complex Gaussian entries, with zero means and unit variance. In this paper we assume that the mobile transmit antennas and the base station antennas have sufficient interelement spacing such that spatial decorrelation between simultaneously transmitted data streams on different transmit channels is feasible. For mobile devices, MIMO-beamforming can usually be accommodated for frequency bands higher than $2.5 \mathrm{GHz}$. However, a new approach is needed to support multiple antennas in the lower frequency ranges due to the highly correlated channels. The transfer functions from the $l_{s}$ th SU device to the SBS antenna array (the cascade of $\mathbf{H}_{s s, l_{s}}[k]$ and $\left.\mathbf{w}_{l_{s}}^{t}[k]\right)$ result in a unique spatial signature for each SU, which can be exploited to affect the separation of the user data at the SBS using appropriate multiuser detection techniques. The SBS detects all $L_{s}$ SUs, simultaneously at the multiuser detection module of the SDMA system, by multiplying the output of the array with the $N_{s}^{r} \times 1$ receiving 


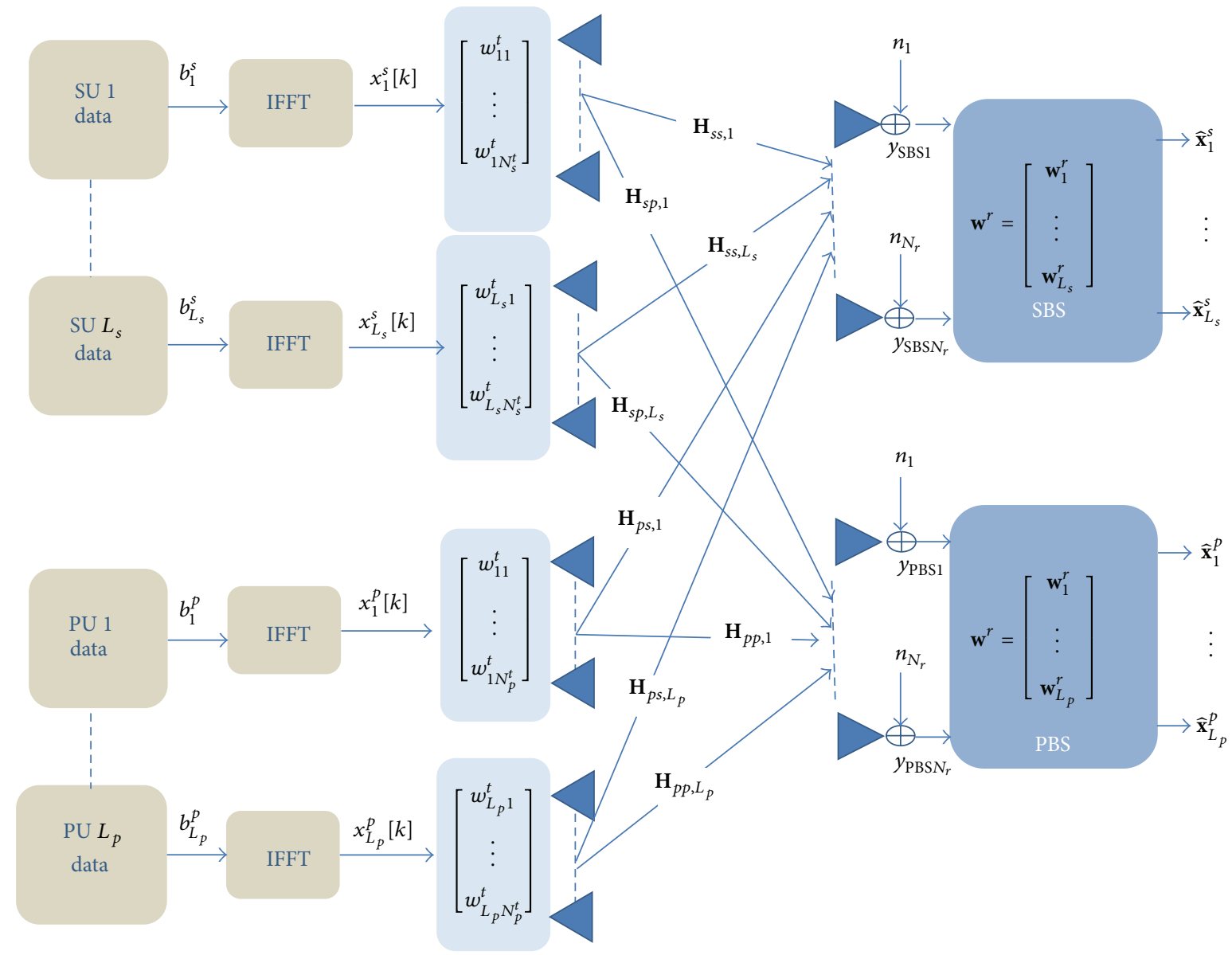

FIGURE 1: System model: primary and secondary OFDM-SDMA systems.

weight vectors, $\mathbf{w}_{l_{s}}^{r}[k]=\left[w_{l_{s} 1}^{r}[k], w_{l_{s} 2}^{t}[k], \ldots, w_{l_{s} N_{s}^{r}}^{r}[k]\right]^{T}$, for each SU $l_{s}$. The detection of secondary user $l_{s}$ out of $L-1$ interfering users $\left(L=L_{s}+L_{p}\right)$ can thus be depicted as

$$
\begin{aligned}
\widehat{\mathbf{x}}_{l_{s}}[k] & =\mathbf{w}_{l_{s}}^{r, H}[k] \mathbf{y}_{\mathrm{SBS}}[k] \\
& =\mathbf{S}_{d}[k]+\mathbf{S}_{I_{s}}[k]+\mathbf{S}_{I_{p}}[k]+\mathbf{N}[k],
\end{aligned}
$$

where $\mathbf{N}[k]=\mathbf{w}_{l_{s}}^{r, H}[k] \mathbf{n}[k]$ is the noise signal at the array output of the SBS, $\mathbf{S}_{d}[k]$ is the desired signal for the detection of user $l_{s}$ 's signal, $\mathbf{S}_{I_{s}}[k]$ is the multiple-access interference (MAI) contributed by the $L_{s}-1$ other SUs, and $\mathbf{S}_{I_{p}}[k]$ is the MAI from $L_{p}$ PUs. $\mathbf{S}_{d}[k], \mathbf{S}_{I_{s}}[k]$, and $\mathbf{S}_{I_{p}}[k]$ are, respectively, given by

$$
\begin{array}{r}
\mathbf{S}_{d}[k]=\mathbf{w}_{l_{s}}^{r, H}[k] \mathbf{H}_{s s, l_{s}}[k] \mathbf{w}_{l_{s}}^{t}[k] \mathbf{x}_{l_{s}}^{s}[k], \\
\mathbf{S}_{I_{s}}[k]=\mathbf{w}_{l_{s}}^{r, H}[k]\left[\sum_{i=1, i \neq l_{s}}^{L_{s}} \widetilde{\mathbf{H}}_{s s, i}^{t}[k] \mathbf{x}_{i}^{s}[k]\right], \\
\mathbf{S}_{I_{p}}[k]=\mathbf{w}_{l_{s}}^{r, H}[k]\left[\sum_{l_{p}=1}^{L_{p}} \widetilde{\mathbf{H}}_{p s, l_{p}}^{t}[k] \mathbf{x}_{l_{p}}^{p}[k]\right],
\end{array}
$$

where $\widetilde{\mathbf{H}}_{s s, l_{s}}^{t}[k]=\mathbf{H}_{s s, l_{p}}[k] \mathbf{w}_{l_{s}}^{t}[k]$ and $\widetilde{\mathbf{H}}_{p s, l_{p}}^{t}[k]=\mathbf{H}_{p s, l_{p}}[k]$ $\mathbf{w}_{l_{p}}^{t}[k]$.
During the analysis, perfect channel estimation is assumed. This assumption is justified by the fact that when the number of antennas grows towards infinity, effects of noise, interference, and imperfect channel state information (CSI) disappear [16-18]. However, when pilot-based CSI is used with time-division duplex (TDD) MIMO systems, pilot contamination remains (PC) a limiting factor [22-25]. PC is a well-known problem in pilot-aided channel estimation and it happens when other users in the system are reusing the same set of pilot signals due to the limitation imposed by the number of available orthogonal pilots. This PC problem is accentuated even more with massive MIMO since it causes the interference rejection performance to quickly saturate with the number of antennas. Within the context of cognitive radio, if there is no cooperation between the $\mathrm{SN}$ and the $\mathrm{PN}$, it is likely that the pilots used in both networks do not satisfy orthogonality, which may lead to pilot contamination. However, with some cooperation between the two networks and under the assumption that the pilots of each network are orthogonal, the SBS and the PBS can efficiently mitigate pilot contamination from PUs and SUs, respectively, by assigning different time to the training phase in both networks. Another alternative, if there is no cooperation between the two networks, is to use blind channel estimation techniques that require no or a minimal number of pilot symbols. One particular class of blind methods that works well with massive 
MIMO is the one based on subspace estimation techniques [26-29] that estimate the channel from the eigenvector of the covariance matrix of the received signal. This class requires unused degrees of freedom, which is the case of massive MIMO where the number of antennas at the base station is much larger than the number of users.

\section{Capacity-Aware MIMO-Beamforming in Cognitive OFDM-SDMA Systems}

Our objective is to find the optimal beamforming vector, $\left(\mathbf{w}_{l_{s}}^{t}[k]\right)_{\text {opt }}$, that maximizes the Ergodic capacity of the cognitive OFDM-SDMA channel for each $\mathrm{SU} l_{s}$ of the $\mathrm{SN}$ imposing the following two sets of constraints: (1) each secondary user $l_{s}$ has a limited maximum transmission power equal to $P_{\max , l}^{t}$ and (2) the total maximum interference power at the PBS from the SUs does not exceed the maximum power constraint of $J_{s p}^{\max }$. In mathematical terms, these two constraints are expressed as follows [30]:

$$
\begin{aligned}
& \max _{\mathbf{w}_{l_{s}}^{t}[k]}\left[E\left(\log _{2}\left\{\mathbf{I}+\frac{\rho_{l_{s}}}{N_{s}^{t}}\left|B_{l_{s}}^{-1 / 2}[k] \widetilde{\mathbf{H}}_{s s, l_{s}}^{t}[k]\right|^{2}\right\}\right)\right] \\
& \text { Subject to : }\left\{\begin{array}{c}
\mathbf{w}_{l_{s}}^{t, H}[k] \mathbf{w}_{l_{s}}^{t}[k] \leq P_{\max , l_{s}}^{t} \\
J_{s p}=\sum_{i=1}^{L_{s}} \widetilde{\mathbf{H}}_{s p, l_{s}}^{t}[k] \widetilde{\mathbf{H}}_{s p, l_{s}}^{t, H}[k] \leq J_{s p}^{\max }
\end{array}\right\},
\end{aligned}
$$

where $E[\cdot]$ denotes the expectation operator, $\widetilde{\mathbf{H}}_{s s, l_{s}}^{t}[k]=$ $\mathbf{H}_{s s, l_{s}}[k] \mathbf{w}_{l_{s}}^{t}[k], \widetilde{\mathbf{H}}_{s p, l_{s}}^{t}[k]=\mathbf{H}_{s p, l_{s}}[k] \mathbf{w}_{l_{s}}^{t}[k], \mathbf{B}_{l_{s}}[k]=\mathbf{B}_{s s}[k]+$ $\mathbf{B}_{p s}[k]+\sigma_{n}^{2} \mathbf{I}_{N_{s}^{r}}[k], \mathbf{B}_{s s}[k]=\sum_{i=1, i \neq l_{s}}^{L_{s}} \widetilde{\mathbf{H}}_{s s, l_{s}}^{t}[k] \widetilde{\mathbf{H}}_{s s, l_{s}}^{t, H}[k]$, and $\mathbf{B}_{p s}[k]=\sum_{l_{p}=1}^{L_{p}} \widetilde{\mathbf{H}}_{p s, l_{s}}^{t}[k] \widetilde{\mathbf{H}}_{p s, l_{s}}^{t, H}[k]$.

This problem is a constrained optimization problem which is highly nonconvex and complicated to solve. However, a suboptimal solution can be obtained by exploiting the method of Lagrange multipliers as follows:

$$
\begin{aligned}
\mathscr{L}_{\mathrm{CCA}}\left(\mathbf{w}_{l_{s}}^{t}, v_{l_{s}}, \lambda_{l_{s}}\right) & \\
= & E\left(\log _{2}\left\{\mathbf{I}+\frac{\rho_{l_{s}}}{N_{s}^{t}}\left|B_{l_{s}}^{-1 / 2}[k] \widetilde{\mathbf{H}}_{s s, l_{s}}^{t}[k]\right|^{2}\right\}\right) \\
& -v_{l_{s}}\left(\frac{\sum_{l_{s}=1}^{L_{s}} \widetilde{\mathbf{H}}_{s p, l_{s}}^{t}[k] \widetilde{\mathbf{H}}_{s p, l_{s}}^{t, H}[k]}{\left.J_{s p}^{\max }-1\right)}\right. \\
& -\lambda_{l_{s}}\left(\frac{\mathbf{w}_{s, l_{s}}^{t, H}[k] \mathbf{w}_{l_{s}}^{t}[k]}{P_{\max , l_{s}}^{t}}-1\right)
\end{aligned}
$$

where $\nu_{l_{s}}$ and $\lambda_{l_{s}}$ are the Lagrange multipliers associated with the $l_{s}$ th SU transmission power and the PBS received interference, respectively. In the proposed cognitive capacity-aware (CCA) algorithm, the weight vector for user $l_{s}$ is updated at each iteration $n$, according to

$$
\mathbf{w}_{l_{s}}^{t, \mathrm{CCA}}(n+1)=\mathbf{w}_{l_{s}}^{t, \mathrm{CCA}}(n)+\mu \nabla_{\mathbf{w}_{l_{s}}^{t, \mathrm{CCA}}} \mathscr{L}_{\mathrm{CCA}}\left(\mathbf{w}_{l_{s}}^{t, \mathrm{CCA}}, \eta_{l_{s}}, \lambda_{l_{s}}\right),
$$

where $\nabla_{\mathbf{w}_{l_{s}}^{t, \text { CCA }}}$ is the gradient of $\mathscr{L}_{\mathrm{CCA}}\left(\mathbf{w}_{l_{s}}^{t, \mathrm{CCA}}, \eta_{l_{s}}, \lambda_{l_{s}}\right)$ with respect to $\mathbf{w}_{l_{s}}^{t, \mathrm{CCA}}$ and $\mu$ is an adaptation constant to be chosen relatively small in order to achieve convergence [31]. Notice that SDMA is subcarrier parallel and that the update is done separately on each subcarrier. For brevity, therefore, we drop the frequency index $[k]$ and concentrate on the iteration index $(n)$ in this recursion. Using the matrix derivative formula $\partial \log |\mathbf{A}+\mathbf{B X C}| / \partial \mathbf{X}^{*}=\left[\mathbf{C}(\mathbf{A}+\mathbf{B X C})^{-\mathbf{1}} \mathbf{B}\right]^{H}[32,33]$, the gradient of the Lagrangian can be expressed as

$$
\begin{aligned}
\nabla_{\mathbf{w}_{l_{s}}^{t}} & \mathscr{L}_{\mathrm{CCA}}\left(\mathbf{w}_{l_{s}}^{t}, \eta_{l_{s}}, \lambda_{l_{s}}\right) \\
= & \left(\frac{1}{\ln 2}\right)\left[\frac{\left(\rho_{l_{s}} B_{l_{s}}^{-1}(n) \mathbf{w}_{l_{s}}^{t}(n)\right)}{\left(1+\rho_{l_{s}} \mathbf{w}_{l_{s}}^{t, H}(n) B_{l_{s}}^{-1}(n) \mathbf{w}_{l_{s}}^{t}(n)\right)}\right] \\
& -\left(\frac{\lambda_{l_{s}}(n)}{P_{\max , l_{s}}^{t}}\right) \mathbf{w}_{l_{s}}^{t}(n)-\left(\frac{\nu_{l_{s}}(n)}{J_{s p}^{\max }}\right) \mathbf{H}_{s p, l_{s}}^{H}(n) \mathbf{H}_{s p, l_{s}}(n) \mathbf{w}_{l_{s}}^{t}(n) .
\end{aligned}
$$

The Lagrange multipliers, $\nu_{l_{s}}(n)$ and $\lambda_{l_{s}}(n)$, are updated iteratively using the subgradient based method as described in [31]

$$
\begin{gathered}
\lambda_{l_{s}}(n+1)=\lambda_{l_{s}}(n)-\alpha_{\lambda_{l_{s}}}\left[\mathbf{w}_{l_{s}}^{t, H}(n+1) \mathbf{w}_{l_{s}}^{t}(n+1)-P_{\max , l_{s}}^{t}\right], \\
v_{l_{s}}(n+1)=v_{l_{s}}(n)-\alpha_{v_{l_{s}}}\left[\sum_{l_{s}=1}^{L_{s}} \widetilde{\mathbf{H}}_{s p, l_{s}}^{t}(n) \widetilde{\mathbf{H}}_{s p, l_{s}}^{t, H}(n)-J_{s p}^{\max }\right],
\end{gathered}
$$

where the parameters $\alpha_{\lambda_{l_{s}}}$ and $\alpha_{v_{l_{s}}}$ are the subgradients' stepsizes, whose values are to be chosen relatively small in order to achieve convergence [31].

In our optimization procedure we consider that the initial value of $\mathbf{w}_{l_{s}}^{t, \mathrm{CCA}}(n)$ and $\mathbf{w}_{l_{s}}^{t, \mathrm{CEBF}}(n)$ at iteration index $n=0$ is given by the eigen-beamforming (EBF) weight; that is, $\mathbf{w}_{l_{s}}^{t, \mathrm{CCA}}(0)=\mathbf{w}_{l_{s}}^{t, \mathrm{CEBF}}(0)=\sqrt{P_{\max , l_{s}}^{\mathbf{t}}} \mathbf{u}_{\max , l_{s}}$, where $\mathbf{u}_{\max , l_{s}}$ denotes the eigenvector corresponding to $\lambda_{\max , l_{s}}$, the maximum eigenvalue of $\mathbf{H}_{s p, l_{s}}^{H} \mathbf{H}_{s s, l_{s}}$. This initial value is then used to compute the initial value of the received beamforming vector at iteration index $n=0$. In our case we assume MRC at the receiving SBS; that is,

$$
\mathbf{w}_{l_{s}}^{r}(0)=\left(\mathbf{B}_{l_{s}}(0)\right)^{-1} \widetilde{\mathbf{H}}_{s s, l_{s}}^{t}(0) .
$$

\section{Symbol Error Rate (SER) Performance of Cognitive OFDM-SDMA Systems}

The symbol error rate, $\mathrm{SER}_{k, l_{s}}$, associated with $K$ th subcarrier of user $l_{s}$, can be expressed as [34]

$$
\operatorname{SER}_{k, l_{s}}=E_{\gamma_{k, l_{s}}}\left[a Q\left(\sqrt{2 b \gamma_{k, l_{s}}}\right)\right]
$$

where $E[\cdot]$ denotes the expectation operator, $Q(\cdot)$ denotes the Gaussian Q-function, $\gamma_{k, l_{s}}$ is the signal-to-interference-plusnoise ratio (SINR) associated with the $K$ th subcarrier of user 
$l_{s}$, and $a$ and $b$ are modulation-specific constants. For binary phase shift keying (BPSK), $a=1$ and $b=1$, and, for binary frequency shift keying (BFSK) with orthogonal signaling, $a=$ 1 and $b=0.5$, while, for M-ary phase shift keying (M-PSK), $a=2$ and $b=\sin ^{2}(\pi / M)$. The SINR for SU $l_{s}$ at iteration $n$, $\gamma_{k, l_{s}}(n)$, is given by

$$
\gamma_{k, l_{s}}(n)=\frac{\left(\mathbf{w}_{l_{s}}^{r}(n)\right)^{H} \widetilde{\mathbf{H}}_{s s, l_{s}}^{t}(n)\left(\widetilde{\mathbf{H}}_{s s, l_{s}}^{t}(n)\right)^{H} \mathbf{w}_{l_{s}}^{r}(n)}{\left(\mathbf{w}_{l_{s}}^{r}(n)\right)^{H} \mathbf{B}_{l_{s}}(n) \mathbf{w}_{l_{s}}^{r}(n)} .
$$

The average $\mathrm{SER}_{l_{s}}$ performance for user $l_{s}$ can be estimated as [6]

$$
\mathrm{SER}_{l_{s}}=\frac{1}{N_{c}} \sum_{k=0}^{N_{c}-1} \mathrm{SER}_{k, l_{s}}
$$

We observe that, in general, the off-diagonal elements of $\mathbf{B}_{l_{s}}(n)$ are nonzero, reflecting the colour of the interference. However, in the asymptotic case of large $N_{s}^{r}$-element array and given equal power transmitted by all users $\left(P_{l_{s}}=P_{s}\right.$ and $P_{l_{p}}=P_{p}$ ), the central limit theorem (CLT) can be invoked to show that [11]

$$
\mathbf{B}_{l_{s}}(n)=\left(\frac{\left(L_{s}-1\right) P_{s}+L_{p} P_{p}}{\sigma_{n}^{2}}+1\right) \sigma_{n}^{2} \mathbf{I}_{N_{s}^{r}} .
$$

Thus, assuming MRC at the receiving SBS, we can express $\gamma_{l_{s}}(n)$ as

$$
\begin{aligned}
\gamma_{l_{s}}^{\mathrm{CCA}}(n)= & \left(\frac{1}{\left(L_{s}-1\right) P_{s}+L_{p} P_{p}+\sigma_{n}^{2}}\right) \\
& \times \mathbf{w}_{l_{s}, \mathrm{CCA}}^{t, H}(n) \mathbf{H}_{s s, l_{s}}^{H}(n) \mathbf{H}_{s s, l_{s}}(n) \mathbf{w}_{l_{s}, \mathrm{CCA}}^{t}(n) .
\end{aligned}
$$

For the case $n=1$,

$$
\begin{aligned}
\mathbf{w}_{l_{s}}^{t, \mathrm{CCA}} & (1) \\
= & \mathbf{w}_{s, l_{s}}^{t, \mathrm{EBF}}+\left(\frac{\mu}{\ln 2}\right) \\
& \times\left[\frac{\left(\rho_{l_{s}} \mathbf{w}_{l_{s}}^{t, \mathrm{EBF}}\right)}{\left(\left(L_{s}-1\right) P_{s}+L_{p} P_{p}+\sigma_{n}^{2}+\rho_{l_{s}} \mathbf{w}_{l_{s}, \mathrm{EBF}}^{t, H} \mathbf{w}_{l_{s}, \mathrm{EBF}}^{t}\right)}\right] \\
& -\mu\left(\frac{\lambda_{l_{s}}(0)}{\left.P_{\max , l_{s}}^{t}\right) \mathbf{w}_{l_{s}, \mathrm{EBF}}^{t}}\right. \\
& -\mu\left(\frac{v_{l_{s}}(0)}{J_{s p}^{\max }}\right) \mathbf{H}_{s p, l_{s}}^{H}(n) \mathbf{H}_{s p, l_{s}}(n) \mathbf{w}_{l_{s}, \mathrm{EBF} \cdot}^{t} .
\end{aligned}
$$

Substituting (18) into (17), we can express $\gamma_{l_{s}}^{\mathrm{CCA}}(n)$ at iteration index $n=1$ as

$$
\gamma_{l_{s}}^{\mathrm{CCA}}(1)=(1+\alpha+\beta+\eta) \gamma_{l_{s}}^{\mathrm{EBF}}
$$

where

$$
\begin{gathered}
\alpha=\left(\frac{2 \mu \kappa_{0}}{\left(L_{s}-1\right) P_{s}+L_{p} P_{p}+\sigma_{n}^{2}}\right. \\
\left.+\frac{2 \mu^{2} \kappa_{0}^{2}}{\left[\left(L_{s}-1\right) P_{s}+L_{p} P_{p}+\sigma_{n}^{2}\right]^{2}}\right), \\
\kappa_{0}=\left(\frac{1}{\ln 2}\right)\left[\frac{\rho_{l_{s}}}{\left(1+P_{s} /\left(\left(L_{s}-1\right) P_{s}+L_{p} P_{p}+\sigma_{n}^{2}\right)\right)}\right], \\
\beta=\left(\frac{\mu \cdot \lambda_{l_{s}}(0)}{P_{\max , l_{s}}^{t}}\right)^{2}, \\
\eta=\left(\frac{\mu \cdot \nu_{l_{s}}(0)}{J_{s p}^{\max }}\right)^{2},
\end{gathered}
$$

and $\gamma_{l_{s}}^{\mathrm{EBF}}$ represents the SINR obtained when eigen-beamforming is used and can be expressed as

$$
\begin{aligned}
\gamma_{l_{s}}^{\mathrm{EBF}} & =\left(\frac{1}{\left(L_{s}-1\right) P_{s}+L_{p} P_{p}+\sigma_{n}^{2}}\right) \mathbf{w}_{l_{s}, \mathrm{EBF}}^{t, H} \mathbf{H}_{s s, l_{s}}^{H} \mathbf{H}_{s s, l_{s}} \mathbf{w}_{l_{s}, \mathrm{EBF}}^{t, H} \\
& =\left(\frac{P_{s}}{\left(L_{s}-1\right) P_{s}+L_{p} P_{p}+\sigma_{n}^{2}}\right) \lambda_{\max , l_{s}}
\end{aligned}
$$

Substituting (21) into (19), then we can write $\gamma_{l_{s}}^{C C A}(1)$ as

$$
\gamma_{l_{s}}^{\mathrm{CCA}}(1)=\left(\frac{(1+\alpha+\beta+\eta) P_{s}}{\left(L_{s}-1\right) P_{s}+L_{p} P_{p}+\sigma_{n}^{2}}\right) \lambda_{\max , l_{s}} .
$$
as [8]

Therefore, an alternate expression for (13) can be written

$$
\begin{aligned}
& \mathrm{SER}_{k, l_{s}} \\
& =\frac{a \sqrt{b}}{2 \sqrt{\pi}} \\
& \quad \times \int_{0}^{\infty} \frac{e^{-b u}}{\sqrt{u}} F_{\lambda_{\max , l_{s}}}\left(\frac{\left[\left(L_{s}-1\right) P_{s}+L_{p} P_{p}+\sigma_{n}^{2}\right] u}{P_{s}(1+\alpha+\beta+\eta)}\right) d u,
\end{aligned}
$$

where $F_{\lambda_{\max , l_{s}}}(u)$ is the cumulative distribution function (cdf) of $\lambda_{\max , l_{s}}$. For the case $N_{s}^{t}=2$ and $N_{s}^{t} \leq N_{s}^{r}, F_{\lambda_{\max , l_{s}}}(u)$ is given by [35]

$$
\begin{aligned}
F_{\lambda_{\max , l_{s}}}(u)= & \frac{\operatorname{det}\left(\mathbf{R}_{s}^{t}\right)}{\Delta_{2}\left(\mathbf{R}_{s}^{t}\right) \Delta_{N_{s}^{r}}\left(\mathbf{R}_{s}^{r}\right)} \sum_{p=1}^{N_{s}^{r}} \sum_{t=1, t \neq p}^{N_{s}^{r}}(-1)^{p+\phi(t)} \\
& \cdot\left(\beta_{p} \beta_{t}\right)^{N_{s}^{r}-1} \Delta_{N_{s}^{r}-2}(A) \Phi_{p, t}(u),
\end{aligned}
$$


where $\Delta_{m}(\mathbf{R})$ is a Vandermonde determinant in the eigenvalues $\left\{\beta_{i}\right\}_{i=1}^{m}$ of the $m$-dimensional matrix argument, given by $\Delta_{m}(\mathbf{R})=\Pi_{i<j}^{m}\left(\beta_{j}-\beta_{i}\right)$, while

$$
\phi(t)= \begin{cases}t, & \text { for } t<p \\ t-1, & \text { for } t>p\end{cases}
$$

and $A=\left\{\beta_{i} ; i \in\left\{1, \ldots, N_{s}^{r}\right\} \backslash\{p, t\}\right\}$. Also $\Phi_{p, t}(u)=$ $(1 / u) e^{-u / \omega_{2} \beta_{p}} \Psi\left(N_{s}^{r} ;-u / \omega_{2} \beta_{p}\right) e^{-u / \omega_{1} \beta_{t}} \Psi\left(N_{s}^{r} ;-u / \omega_{1} \beta_{t}\right)$, where $\Psi(l ; y)=1-e^{-y} \sum_{k=0}^{l-1} y^{k} / k !$ is the regularized lower incomplete gamma function. Next, we substitute (24) into (23) and we consider high SNR case; then the SER can be expressed asymptotically as [8]

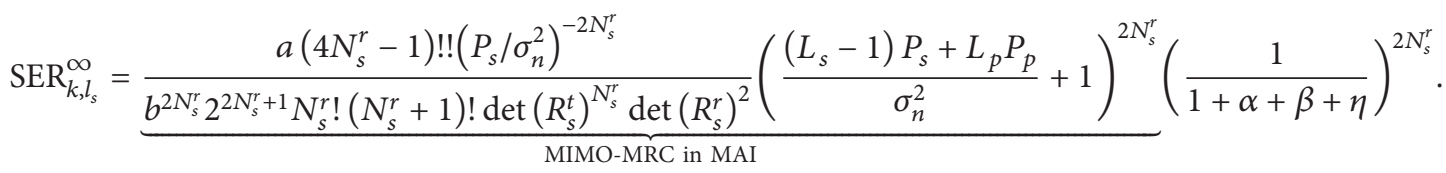

The first term represents the MIMO-MRC system, where the SER performance is mainly dominated by the multiuser access interference (MAI), under transmitted power constraints. It is noted that the presence of PUs' interferences contributes asymptotically an SER degradation of $\left(L_{p} P_{p} /\left[\left(L_{s}-1\right) P_{s}+\sigma_{n}^{2}\right]+1\right)^{2 N_{s}^{r}}$ compared to the system without PUs. This degradation is for each SU and in each OFDM subcarrier. On the other hand, we observe that, in the asymptotic case of large $N_{s}^{r}$-element, the additional factor in the SER expression of the proposed scheme, $(1 /[1+\alpha+\beta+\eta])^{2 N_{s}^{r}}$, will approach zero, since $\alpha \geq 0, \beta \geq$ 0 , and $\eta \geq 0$. Therefore, the proposed adaptive MIMObeamforming scheme, in addition to protecting the primary network, can improve the SER performance of cognitive OFDM-SDMA multiuser access systems compared to conventional MIMO-MRC that suffers an error floor in the SER performance due to MAI. It is also noted that at the asymptotic case of large number of base station antennas and high SNR the SER performances are almost independent of the number of transmitting antennas. Next, we examine the total interference power at the PBS from the SUs when using CCA. Substituting (18) into (4) and after some manipulations, we can express $J_{s p}^{\mathrm{CCA}}$ at iteration index $n=1$ as

$$
\begin{aligned}
J_{s p}^{\mathrm{CCA}} & =L_{s} P_{s}(C-K)\left(C \beta_{l_{s}, \max }-K\right) \\
& =J_{s p}^{\mathrm{EBF}}(C-K)\left(C \beta_{l_{s}, \max }-K\right),
\end{aligned}
$$

where $K=(\mu / \ln 2)\left[\left(\rho_{l_{s}} \mathbf{w}_{s, l_{s}}^{t, \mathrm{EBF}}\right) /\left(\left(L_{s}-1\right) P_{s}+L_{p} P_{p}+\sigma_{n}^{2}+\right.\right.$ $\left.\left.P_{s} \rho_{l_{s}}\right)\right]-\mu\left(\lambda_{l_{s}}(0) / P_{\max , l_{s}}^{t}\right)-1, C=\mu\left(\nu_{l_{s}}(0) / J_{s p}^{\max }\right), \beta_{l_{s}, \max }$ is the maximum eigenvalue of $\mathbf{H}_{s p, l_{s}}^{H} \mathbf{H}_{s p, l_{s}}$, and $J_{s p}^{\mathrm{EBF}}=L_{s} P$ is the total interference power at the PBS from the SUs when using EBF. Since the term $(C-K)\left(C \beta_{l_{s}, \text { max }}-K\right) \leq 1$, the total interference power at the PBS from the SUs with the transmit weights obtained using the proposed CCA is better controlled and less than the one based on conventional eigenbeamforming.

\section{Simulation Results}

In our simulation setups we consider a CR-based OFDMSDMA system with $N_{s}^{t}=N_{p}^{t}=2$ transmit antennas. The number of antennas at the PBS and at the SBS is the same, $N_{p}^{r}=N_{s}^{r}$, and varies from 8 up to $24 . L_{s}=4$ SUs and $L_{p}=4$ PUs. We assume BPSK modulation. We impose $P_{\max , l}^{t}=0 \mathrm{~dB}$ and $J_{s p}^{\max }=-5,-10$, and $-15 \mathrm{~dB}$ on the SUs. For OFDM configurations, we assume the 256-OFDM system $\left(N_{c}=256\right)$ which is widely deployed in broadband wireless access services and we ran for 50 iterations with $\mu=0.001$. For the PN we assume an OFDM-SDMA system with the conventional MIMO-MRC, that is, EBF at the transmitter and $\mathrm{MRC}$ at the receiving PBS.

Figure 2 compares the SER performance of SUs achieved by the proposed CCA and the conventional MIMO-MRC for $J_{s p}^{\max }=-15 \mathrm{~dB}$ and $N_{p}^{r}=N_{s}^{r}=8,16$, and 24. It is observed from the results that when $N_{s}^{r}$ increases, the SER of both methods decreases, but the capacity-awarebased method achieves significantly better performance, as expected from the analysis. It is also noted that as $N_{s}^{r}$ increases the performance gap between the two schemes increases.

Figure 3, on the other hand, shows the impact of the interference power constraints, $J_{s p}^{\max }$, on the SER performance of PUs. For both cases, $N_{p}^{r}=N_{s}^{r}=16$ and $N_{p}^{r}=N_{s}^{r}=24$, it is noted that, as $J_{s p}^{\max }$ becomes larger, the SER of PUs is improved due to the decreased interferences. It is also noted that the most significant interference at the PBS is caused when the SN is using the nonconstrained MIMO-MRC scheme, because it is designed without considering interference to PBS. This shows that when $\mathrm{SN}$ is using the proposed cognitive capacityaware scheme, it could efficiently control the interference to PBS. It is also noted that when $N_{p}^{r}=N_{s}^{r}=24$, the SER performance of PUs varies slightly with $J_{s p}^{\max }$ variations. This means that when the number of base station antennas becomes large, the interference constrained imposed on SUs could be relaxed without impacting the SER performance of PUs.

\section{Conclusion}

This paper presents a new adaptive beamforming algorithm for multiuser access in large scale cognitive OFDM-SDMA systems. The proposed algorithm iteratively seeks the optimal transmit weight vectors that maximize the channel capacity 


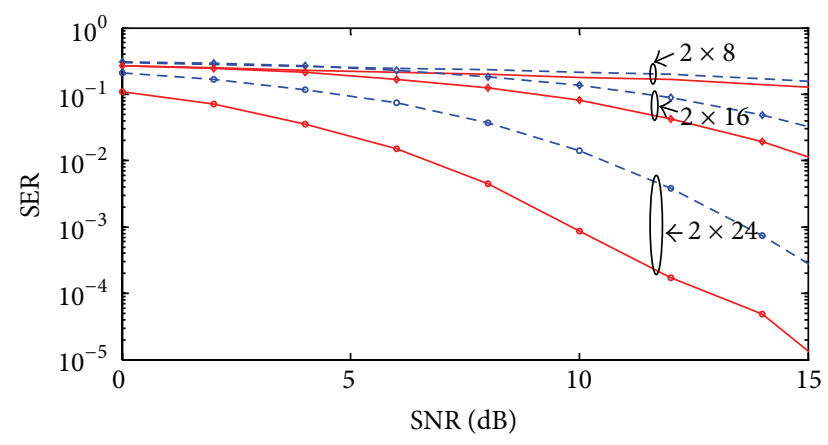

- SER of SN using CCA scheme; $2 \times 8$

- - - SER of SN using MIMO-MRC; $2 \times 8$

$-\diamond-$ SER of SN using MIMO-MRC; $2 \times 16$

$\rightarrow$ SER of SN using CCA scheme; $2 \times 16$

$\rightarrow$ SER of SN using CCA scheme; $2 \times 24$

$-\ominus-$ SER of SN using MIMO-MRC; $2 \times 24$

FIGURE 2: SER performance of SN using CCA and MIMO-MRC schemes for $J_{s p}=-15 \mathrm{~dB}$.

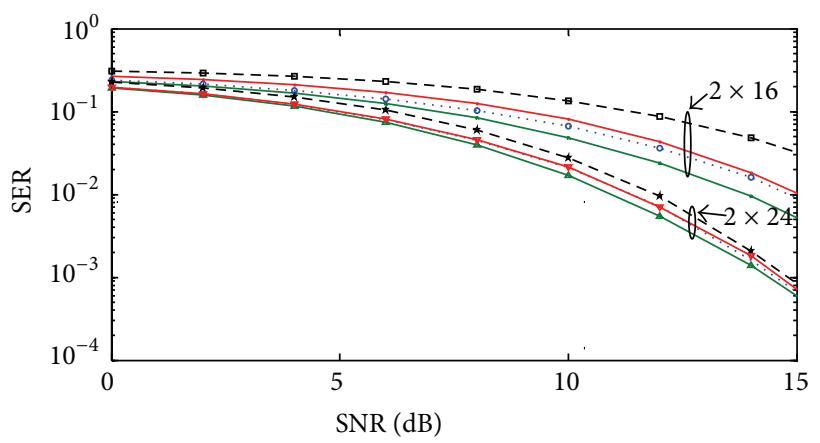

- - SER of PN when SN is using MIMO-MRC; $2 \times 16$

$\ldots$ SER of PN when SN is using CCA; $2 \times 16 ; J_{s p}=-5 \mathrm{~dB}$

\# SER of PN when SN is using CCA; $2 \times 16 ; J_{s p}=-10 \mathrm{~dB}$

o. SER of PN when SN is using CCA; $2 \times 16 ; J_{s p}=-15 \mathrm{~dB}$

$\triangle$ SER of PN when SN is using MIMO-MRC; $2 \times 24$

- - SER of PN when SN is using CCA; $2 \times 24 ; J_{s p}=-5 \mathrm{~dB}$

.... SER of PN when SN is using CCA scheme; $2 \times 24 ; J_{s p}=-10 \mathrm{~dB}$

$\rightarrow$ SER of PN when SN is using CCA; $2 \times 24 ; J_{s p}=-15 \mathrm{~dB}$

FIgURE 3: Impact of $J_{s p}^{\max }$ on the SER of PUs when the SN is using CCA and MIMO-MRC schemes.

of each secondary user in the network while protecting PUs from SUs' interferences. It is shown that the proposed large scale CCA scheme could efficiently control the interference to PBS and could relax the constraints imposed on conventional CR networks.

\section{Conflict of Interests}

The author declares that there is no conflict of interests regarding the publication of this paper.

\section{References}

[1] J. Mitola III and G. Q. Maguire Jr., "Cognitive radio: making software radios more personal," IEEE Personal Communications, vol. 6, no. 4, pp. 13-18, 1999.

[2] S. Haykin, "Cognitive radio: brain-empowered wireless communications," IEEE Journal on Selected Areas in Communications, vol. 23, no. 2, pp. 201-220, 2005.

[3] M. Y. Alias, A. K. Samingan, S. Chen, and L. Hanzo, "Multiple antenna aided OFDM employing minimum bit error rate multiuser detection," Electronics Letters, vol. 39, no. 24, pp. 17691770,2003

[4] P. Vandenameele, L. Van Der Perre, M. G. E. Engels, B. Gyselinckx, and H. J. De Man, "Combined OFDM/SDMA approach," IEEE Journal on Selected Areas in Communications, vol. 18, no. 11, pp. 2312-2321, 2000.

[5] M. Jiang, S. X. Ng, and L. Hanzo, "Hybrid iterative multiuser detection for channel coded space division multiple access OFDM systems," IEEE Transactions on Vehicular Technology, vol. 55, no. 1, pp. 115-127, 2006.

[6] M. Munster and L. Hanzo, "Performance of SDMA multiuser detection techniques for Walsh-Hadamard-spread OFDM schemes," in Proceedings of the 54th IEEE Vehicular Technology Conference (VTC '01), vol. 4, pp. 2319-2323, October 2001.

[7] K. K. Wong, R. Cheng, K. B. Letaief, and R. D. Murch, "Adaptive antennas at the mobile and base stations in an OFDM/TDMA system," IEEE Transactions on Communications, vol. 49, no. 1, pp. 195-206, 2001.

[8] M. Kang and M. Alouini, "A comparative study on the performance of MIMO MRC systems with and without cochannel interference," IEEE Transactions on Communications, vol. 52, no. 8, pp. 1417-1425, 2004.

[9] A. I. Sulyman and M. Hefnawi, "Adaptive MIMO beamforming algorithm based on gradient search of the channel capacity in OFDM-SDMA systems," IEEE Communications Letters, vol. 12, no. 9, pp. 642-644, 2008.

[10] M. Hefnawi, "Space division multiplexing access aided cognitive radio networks," in Proceedings of the 26th Queen's Biennial Symposium on Communications (QBSC '12), pp. 10-14, May 2012.

[11] L. Yang and L. Wang, "Zero-forcing and minimum meansquare error multiuser detection in generalized multicarrier DS-CDMA systems for cognitive radio," Eurasip Journal on Wireless Communications and Networking, vol. 2008, Article ID 541410, 2008.

[12] K. Hamdi, W. Zhang, and K. B. Letaief, "Opportunistic spectrum sharing in cognitive MIMO wireless networks," IEEE Transactions on Wireless Communications, vol. 8, no. 8, pp. 4098-4109, 2009.

[13] R. Zhang and Y.-C. Liang, "Exploiting multi-antennas for opportunistic spectrum sharing in cognitive radio networks," IEEE Journal on Selected Topics in Signal Processing, vol. 2, no. 1, pp. 88-102, 2008.

[14] S. Yiu, M. Vu, and V. Tarokh, "Interference reduction by beamforming in cognitive networks," in Proceedings of the IEEE Global Telecommunications Conference (GLOBECOM '08), pp. 1-6, New Orleans, La, USA, December 2008.

[15] L. Bixio, G. Oliveri, M. Ottonello, and C. S. Regazzoni, "Cognitive radios with multiple antennas exploiting spatial opportunities," IEEE Transactions on Signal Processing, vol. 58, no. 8, pp. 4453-4459, 2010. 
[16] T. L. Marzetta, "Noncooperative cellular wireless with unlimited numbers of base station antennas," IEEE Transactions on Wireless Communications, vol. 9, no. 11, pp. 3590-3600, 2010.

[17] F. Rusek, D. Persson, B. Lau et al., "Scaling up MIMO: opportunities and challenges with very large arrays," IEEE Signal Processing Magazine, vol. 30, no. 1, pp. 40-60, 2013.

[18] J. Hoydis, S. ten Brink, and M. Debbah, "Massive MIMO in the UL/DL of cellular networks: how many antennas do we need?" IEEE Journal on Selected Areas in Communications, vol. 31, no. 2, pp. 160-171, 2013.

[19] H. Bölcskei and A. J. Paulraj, "Performance of space-time codes in the presence of spatial fading correlation," in Proceedings of the 34th Asilomar Conference, pp. 687-693, November 2000.

[20] M. Kang and M.-S. Alouini, "Impact of correlation on the capacity of MIMO channels," in Proceedings of the IEEE International Conference on Communications (ICC '03), vol. 4, pp. 2623-2627, Paris, France, May 2003.

[21] P. J. Smith, S. Roy, and M. Shafi, "Capacity of MIMO systems with semicorrelated flat fading," IEEE Transactions on Information Theory, vol. 49, no. 10, pp. 2781-2788, 2003.

[22] J. Jose, A. Ashikhmin, T. L. Marzetta, and S. Vishwanath, "Pilot contamination and precoding in multi-cell TDD systems," IEEE Transactions on Wireless Communications, vol. 10, no. 8, pp. 2640-2651, 2011.

[23] J. Jose, A. Ashikhmin, T. L. Marzetta, and S. Vishwanath, "Pilot contamination problem in multi-cell TDD systems," in Proceedings of the IEEE International Symposium on Information Theory (ISIT '09), pp. 2184-2188, Seoul, Republic of Korea, July 2009.

[24] H. Q. Ngo, T. L. Marzetta, and E. G. Larsson, "Analysis of the pilot contamination effect in very large multicell multiuser MIMO systems for physical channel models," in Proceedings of the 36th IEEE International Conference on Acoustics, Speech, and Signal Processing (ICASSP '11), pp. 3464-3467, Prague, Czech Republic, May 2011.

[25] B. Gopalakrishnan and N. Jindal, "An analysis of pilot contamination on multi-user mimo cellular systems with many antennas," in Proceedings of the IEEE 12th International Workshop on Signal Processing Advances in Wireless Communications (SPAWC '11), pp. 381-385, San Francisco, Calif, USA, June 2011.

[26] A.-J. van der Veen, S. Talwar, and A. Paulraj, "A subspace approach to blind space-time signal processing for wireless communication systems," IEEE Transactions on Signal Processing, vol. 45, no. 1, pp. 173-190, 1997.

[27] H. Q. Ngo and E. G. Larsson, "EVD-based channel estimation in multicell multiuser MIMO systems with very large antenna arrays," in Proceedings of the IEEE International Conference on Acoustics, Speech, and Signal Processing (ICASSP '12), pp. 32493252, Kyoto, Japan, March 2012.

[28] E. Beres and R. Adve, "Blind channel estimation for orthogonal STBC in MISO systems," IEEE Transactions on Vehicular Technology, vol. 56, no. 4, pp. 2042-2050, 2007.

[29] B. Muquet, M. De Courville, and P. Duhamel, "Subspace-based blind and semi-blind channel estimation for OFDM systems," IEEE Transactions on Signal Processing, vol. 50, no. 7, pp. 16991712, 2002.

[30] S. Boyd and L. Vandenberghe, Convex Optimization, Cambridge University Press, Cambridge, UK, 2004.

[31] E. Mijangos, "Approximate subgradient methods for lagrangian relaxation on networks," in System Modeling and Optimization, vol. 312, pp. 370-381, IFIP International Federation for Information Processing, 2007.
[32] S. Ye and R. S. Blum, "Optimized signaling for MIMO interference systems with feedback," IEEE Transactions on Signal Processing, vol. 51, no. 11, pp. 2839-2848, 2003.

[33] A. Hjorungnes and D. Gesbert, "Complex-valued matrix differentiation: techniques and key results," IEEE Transactions on Signal Processing, vol. 55, no. 6, pp. 2740-2746, 2007.

[34] J. G. Proakis, Digital Communications, McGraw-Hill, New York, NY, USA, 4th edition, 2001.

[35] M. R. McKay, A. J. Grant, and I. B. Collings, "Performance analysis of MIMO-MRC in double-correlated Rayleigh environments," IEEE Transactions on Communications, vol. 55, no. 3, pp. 497-507, 2007. 

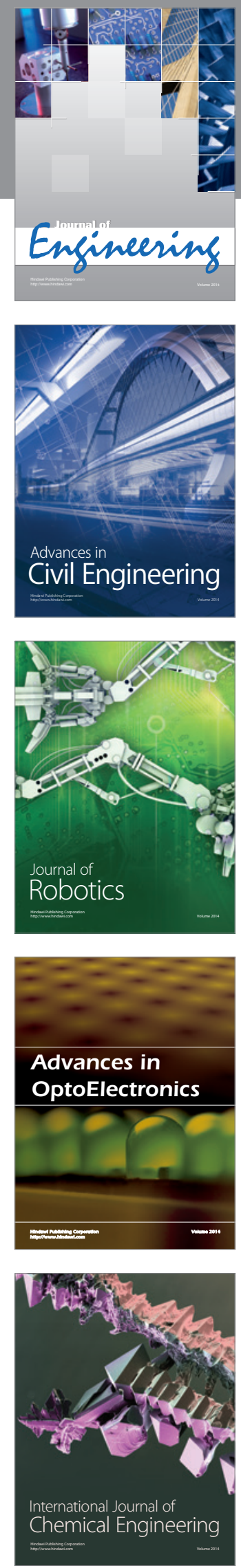

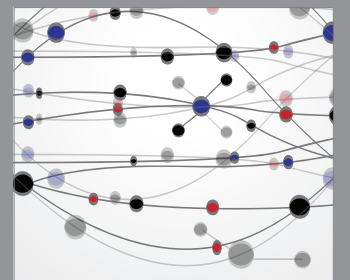

The Scientific World Journal
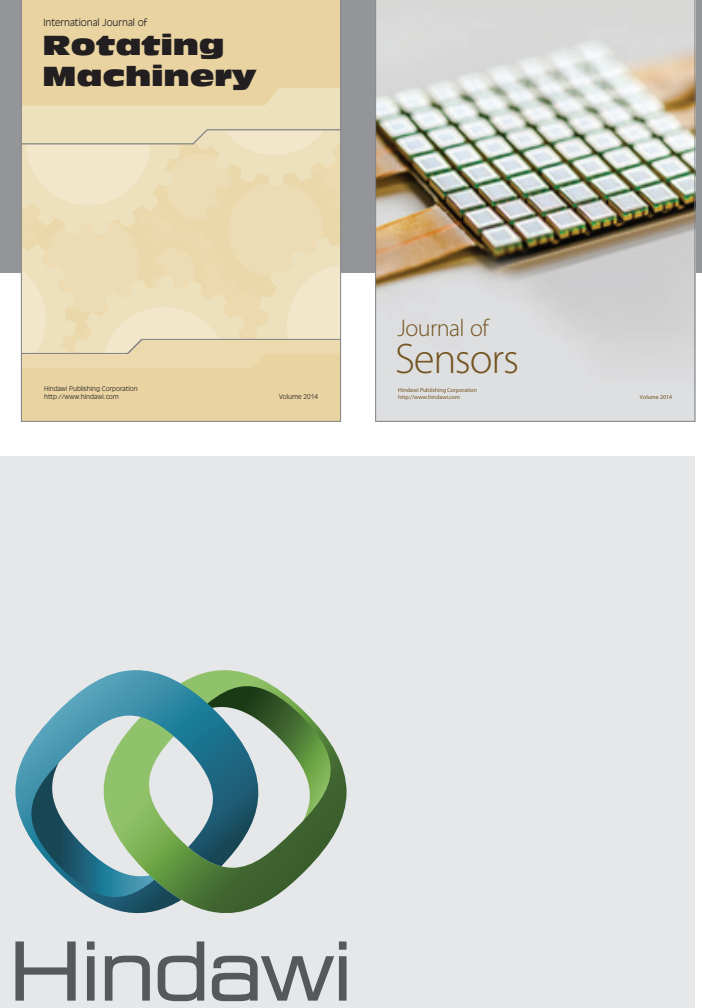

Submit your manuscripts at http://www.hindawi.com
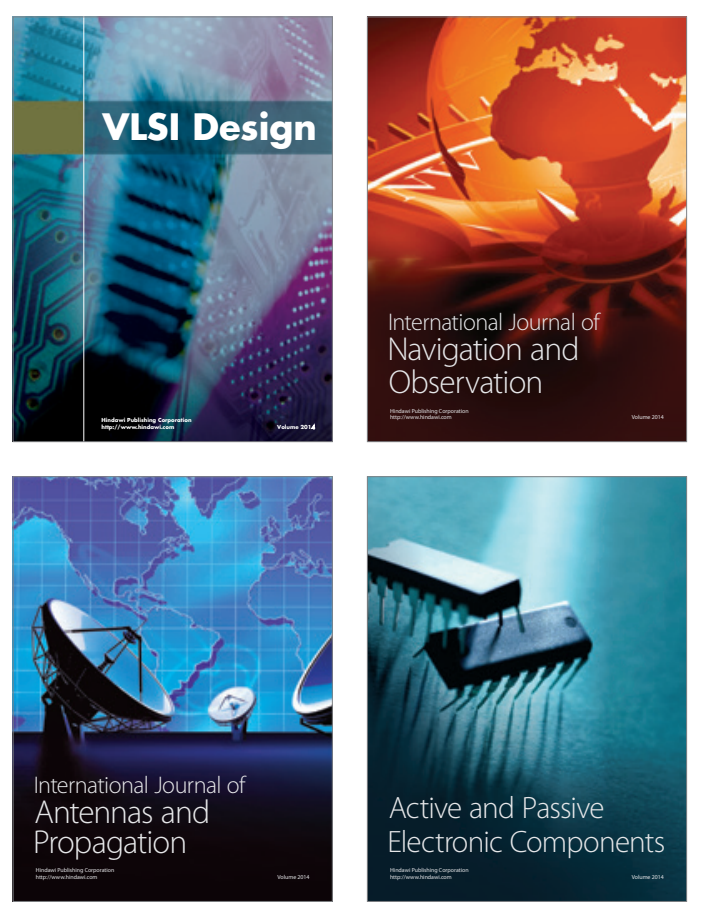
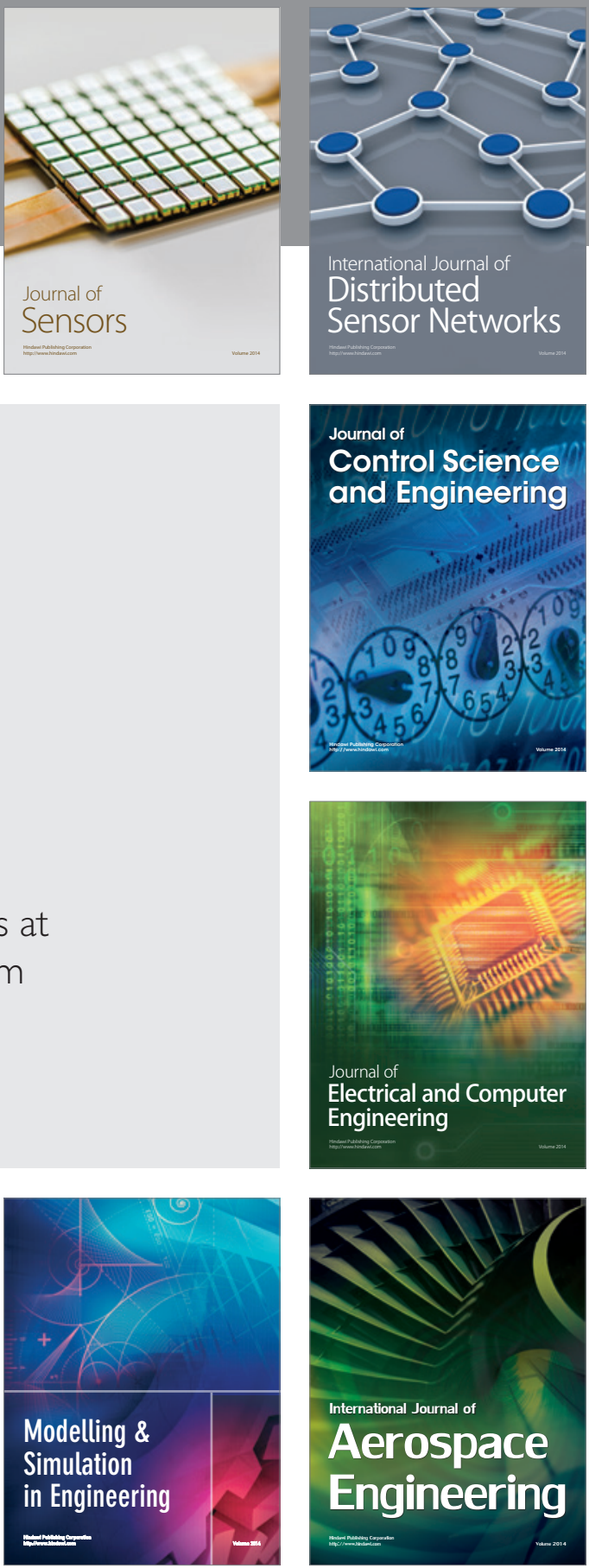

Journal of

Control Science

and Engineering
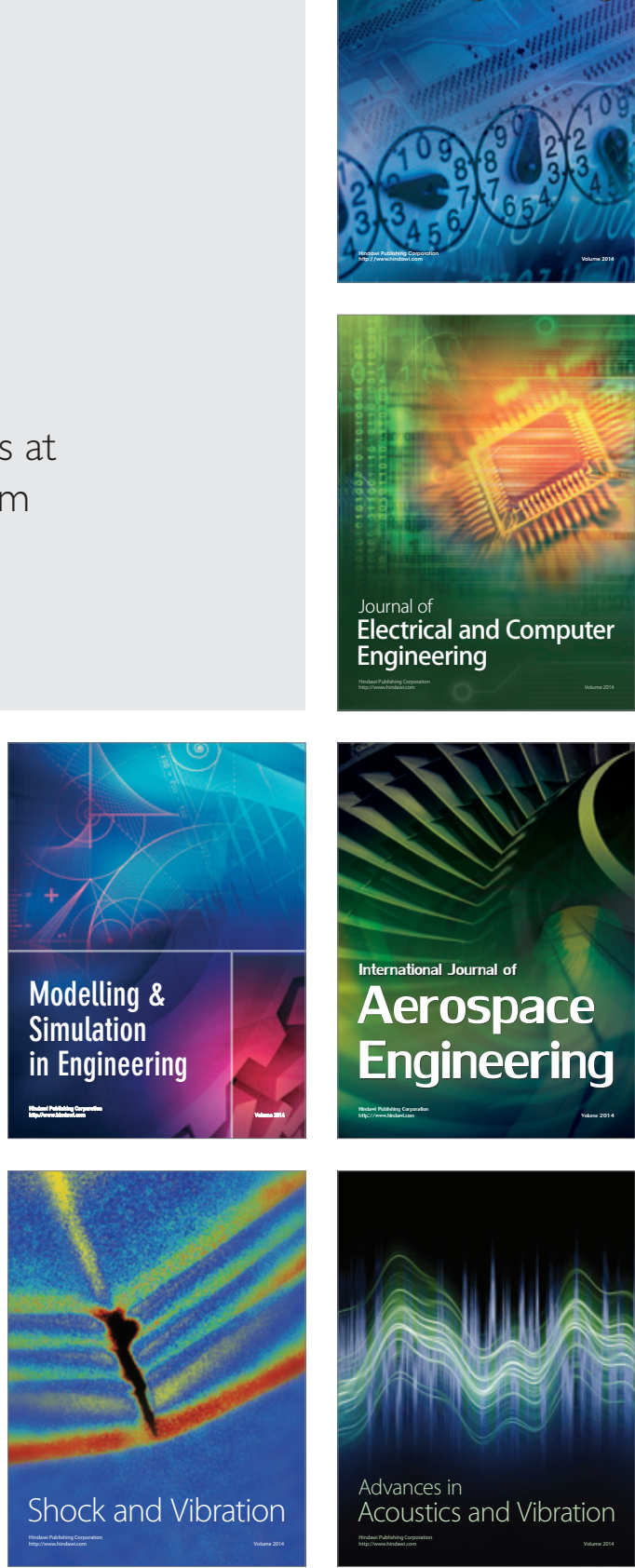